\title{
Diagnóstico e tratamento de infecções sexualmente transmissíveis realizados por enfermeiros na Atenção Primária à Saúde
}

\author{
Diagnosis and Treatment of Sexually Transmitted Diseases performed by nurses in Primary \\ Health Care
}

\section{Diagnóstico y Tratamiento de Infecciones de Transmisión Sexual realizados por enfermeros de Atención Primaria de Salud}

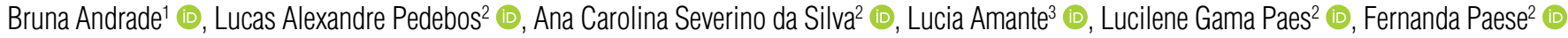 \\ 1 Universidade Estadual de Santa Catarina, Escola de Saúde Pública de Florianópolis - Florianópolis (SC), Brasil. \\ 2Prefeitura de Florianópolis, Secretaria Municipal de Saúde - Florianópolis (SC), Brasil. \\ ${ }^{3}$ Universidade Federal de Santa Catarina, Centro de Ciências da Saúde - Florianópolis (SC), Brasil.
}

\section{Resumo}

Introdução: No final da década de 1990, iniciou-se as atividades de prevenção das infecções sexualmente transmissíveis para a atenção primária à saúde. Nas duas últimas décadas, vem ocorrendo uma ampliação da assistência de enfermeiros no tratamento dessas infecções. A Lei do Exercício Profissional da Enfermagem no 7.498./86, garante aos enfermeiros na consulta de enfermagem o direito de realizar a prescrição de medicamentos aprovados por protocolos institucionais. Objetivo: Verificar se os enfermeiros que atuam na Atenção Primária à Saúde de Florianópolis se sentem aptos para a execução do diagnóstico e do tratamento medicamentoso das infecções sexualmente transmissíveis (IST) com base nos Protocolos de Enfermagem do município. Métodos: Pesquisa exploratório-descritiva de abordagem qualitativa realizada por meio de entrevista semiestruturada com perguntas disparadoras. Foram entrevistados dez enfermeiros assistenciais da Atenção Primária à Saúde do município. Os dados foram analisados segundo a técnica de Bardin. Resultados: Identificaram-se duas categorias analíticas: "O enfermeiro e a (re) construção de sua prática profissional" e "O enfermeiro e a sua contribuição na qualidade de serviço na Atenção Primária à Saúde". Conclusões: Os enfermeiros da rede assistencial da Atenção Primária à Saúde de Florianópolis mostram-se altamente qualificados e sentem-se capazes de responder adequadamente às necessidades de sua prática profissional.

Palavras-chave: Educação em enfermagem; Enfermagem familiar; Doenças sexualmente transmissíveis; Atenção Primária à Saúde; Enfermagem em saúde comunitária; Enfermagem em saúde pública.
*Como citar: Andrade B, Pedebos LA, Silva ACS, Amante LN, Paes LG, Paese F. Diagnóstico e tratamento de infecções sexualmente transmissíveis realizados por enfermeiros na Atenção Primária à Saúde. Rev Bras Med Fam Comunidade. 2022;17(44):2755. https://doi.org/10.5712/rbmfc17(44)2755

\author{
Autor correspondente: \\ Bruna Andrade \\ E-mail: bruna.andrade1@gmail.com \\ Fonte de financiamento: \\ não se aplica. \\ Parecer CEP: \\ (CAAE) 15390819.4.0000.0118, \\ CEP/UDESC 3.672 .925 \\ Procedência: \\ não encomendado. \\ Avaliação por pares: \\ externa. \\ Recebido em: 19/10/2020. \\ Aprovado em: 10/10/2021. \\ Editor Associado: \\ Leandro David Wenceslau
}




\begin{abstract}
Introduction: In the 1990s, sexually transmitted infections prevention activities for primary health care began. In the last two decades, there has been an increase in the assistance provided by nurses in the treatment of these infections. The Nusing Professional Practice Law No. $7.498 / 86$ guarantees nurses in consultations the right to prescribe medication approved by institutional protocols. Objective: Identify whether nurses from the Primary Health Care Network in Florianopolis feel able to diagnose and prescribe medications for the treatment of sexually transmitted infections, based on the municipality's nursing protocols. Methods: exploratory-descriptive research with a qualitative approach, conducted through semi-structured interviews with trigger questions. 10 nurses with experience in primary care in the municipality were interviewed. The data were analyzed using the Bardin technique. Results: two analytical categories were identified: The nurse and the (re)construction of their professional practice and the nurse and their contribution to the quality of service in Primary Health Care. Conclusions: The Primary Health Care Network in Florianopolis is highly qualified, and nurses feel capable of adequately responding to the needs of their professional practices.
\end{abstract}

Keywords: Education, nursing; Family nursing; Sexually transmitted diseases; Primary health care; Community health nursing; Public health nursing.

\title{
Resumen
}

Introducción: A fines de la década de 1990 se iniciaron las actividades de prevención de infecciones de transmisión sexual para la atención primaria de salud. En las últimas dos décadas se ha incrementado la asistencia brindada por enfermeras en el tratamiento de estas infecciones. La Ley de Práctica Profesional de Enfermería no 7.498/86 le garantiza al enfermero en las consultas de enfermera el derecho a prescribir medicamentos aprobados por protocolos institucionales. Objetivo: Identificar si los enfermeros de la Red de Atención Primaria de Salud de Florianópolis se sienten capaces de diagnosticar y recetar medicamentos para el tratamiento de infecciones de transmisión sexual, con base en los protocolos de enfermería del municipio. Métodos: investigación exploratoria-descriptiva con enfoque cualitativo, realizada a través de entrevistas semiestructuradas con preguntas gatillo. Se entrevistó a 10 enfermeras con experiencia en atención primaria en el municipio. Los datos se analizaron mediante la técnica de Bardin. Resultados: se identificaron dos categorías analíticas: El enfermero y la (re) construcción de su práctica profesional y el enfermero y su contribución a la calidad del servicio en Atención Primaria de Salud. Conclusiones: La Red de Atención Primaria de Salud de Florianópolis, está altamente calificada y el enfermero se siente capaz de responder adecuadamente a las necesidades de práctica profesional.

Palabras-clave: Educación en enfermería; Enfermería de la Familia; Enfermedades de transmisión sexual; Atención primaria de salud; Enfermería en salud comunitaria; Enfermería en salud pública.

\section{INTRODUÇÃO}

As infecções sexualmente transmissíveis (IST) são consideradas um problema de saúde pública. Segundo a Organização Mundial da Saúde (OMS), estima-se a ocorrência de mais de 1 milhão de contaminações por dia em todo o mundo. ${ }^{1}$

O não tratamento ou o tratamento inadequado das IST traz consequências tais como doença inflamatória pélvica (DIP), gravidez ectópica, infertilidade, cânceres, prematuridade, natimortalidade, mortalidade neonatal e infecções congênitas, além de aumentar o risco de transmissão do vírus da imunodeficiência humana (human immunodeficiency virus - HIV), causador da síndrome da imunodeficiência adquirida (SIDA, do inglês acquired immunodeficiency syndrome - AIDS). ${ }^{2}$

A sifilis é uma das IST mais comuns do mundo, com cerca de 6 milhões de novos casos a cada ano. ${ }^{3}$ Em 2018, no estado de Santa Catarina, foram notificados os totais de 12.138 casos de sífilis adquirida, 2.427 casos de sífilis em gestante, 680 casos de sífilis congênita e 28 óbitos por sífilis congênita. A região da grande Florianópolis tem a segunda maior taxa de detecção de sífilis do estado, com 249,7 casos para cada 100 mil habitantes, e a quarta maior taxa de sífilis congênita, com 11,4 casos para cada mil nascidos vivos. ${ }^{3}$

Ainda em 2018, Santa Catarina ocupou a $11^{a}$ posição no ranking nacional dos estados com maior incidência de HIVIAIDS. Florianópolis apresenta a segunda maior taxa de detecção de HIV em gestantes e está na sexta posição do ranking das capitais com maior incidência de HIVIAIDS. ${ }^{4}$ 
Durante a década de 1980, o Ministério da Saúde (MS) implantou os Centros de Testagem e Aconselhamento (CTA) com o objetivo de diagnosticar IST e aconselhar os indivíduos de maneira ética e resolutiva. Nos anos 1990 essas ações foram compartilhadas com a Atenção Primária à Saúde (APS) ${ }^{5}$ e, nas duas últimas décadas, ocorreu uma ampliação da assistência dos profissionais enfermeiros de forma a inserir esta categoria no tratamento das IST. ${ }^{6}$

ALei do Exercício Profissional da Enfermagem - lei $n^{\circ} 7.498 / 86^{7}$ — garante aos enfermeiros, na consulta de Enfermagem, fazer a prescrição de medicamentos aprovados por protocolos institucionais. Nesse mesmo sentido, a Portaria $n^{\circ}$ 2.436/2017, que aprova a Política Nacional de Atenção Básica (PNAB), estabelece entre as atribuições do enfermeiro a realização da consulta de Enfermagem e seus desdobramentos incluindo a solicitação de exames complementares e a prescrição de medicações conforme protocolos, diretrizes clínicas e terapêuticas ou outras normativas técnicas estabelecidas pelo gestor federal, estadual, municipal ou do Distrito Federal, desde que observadas as disposições legais da profissão. ${ }^{8}$

É necessário que o profissional enfermeiro realize ações de aconselhamento durante as consultas de enfermagem objetivando, entre outros cuidados, a detecção precoce das IST, o tratamento do indivíduo portador e de suas parcerias para prevenir complicações advindas das infecções quando não tratadas ou inadequadamente tratadas. ${ }^{9}$

Com este estudo, pretende-se discutir a inserção dos enfermeiros na APS para o enfrentamento das IST enquanto problema de saúde pública, durante as consultas de enfermagem. O objetivo da pesquisa é verificar se os enfermeiros atuantes na APS de Florianópolis se sentem aptos a diagnosticar as IST e a prescrever medicamentos para tratá-las com base nos Protocolos de Enfermagem do município. A adoção de protocolos clínicos que habilitem o enfermeiro para a realização de diagnóstico e tratamento das IST ainda é um assunto incipiente no país, o que justifica a importância deste trabalho.

\section{MÉTODOS}

Pesquisa descritivo-exploratória, com abordagem qualitativa, realizada por meio de entrevistas com enfermeiros que trabalham em centros de saúde de Florianópolis realizadas no mês de novembro de 2019. As entrevistas duraram, em média, 15 min cada.

O município de Florianópolis conta com 50 centros de saúde, cujo modelo assistencial se organiza com base na Estratégia Saúde da Família (ESF), regulamentada pela Portaria SS/GAB n 283/2007. ${ }^{10}$

A Comissão Permanente de Sistematização da Assistência de Enfermagem (CSAE) possui uma subcomissão dedicada à construção e à revisão dos Protocolos de Enfermagem de Florianópolis. Esta foi criada em 2015 com o objetivo de ampliar a clínica do enfermeiro. Essa subcomissão é formada por enfermeiros da rede municipal de saúde dos cinco Distritos Sanitários atuantes nas equipes de saúde da família, policlínicas, centros de atenção psicossocial (CAPS), unidades de pronto atendimento (UPA), Vigilância em Saúde e Gestão. O trabalho dessa subcomissão é embasado nas melhores evidências disponíveis e seus participantes são responsáveis pela análise dos conteúdos que compõem os protocolos, pela revisão dos protocolos vigentes e pela construção de novos Protocolos de Enfermagem. ${ }^{11}$

Atualmente, a rede da APS de Florianópolis conta com 198 enfermeiros efetivos, distribuídos nas funções de assistência, coordenação e residência. Para a participação neste estudo, o critério de inclusão foi a atuação como enfermeiro assistencial na APS. Alguns dos participantes acumulam cargo de coordenação, sendo esta atribuição simultânea às atividades na assistência. Residentes de Enfermagem em Saúde da Família foram excluídos da amostra. 
A coleta de dados foi realizada por meio de entrevista semiestruturada, composta de questões relativas às práticas dos enfermeiros voltadas para as IST. O questionário foi composto das seguintes perguntas: "Conte-me sobre sua prática de prescrição medicamentosa relacionada ao Protocolo Infecções Sexualmente Transmissíveis e Outras Doenças Transmissíveis de Interesse em Saúde Coletiva (dengue/ tuberculose)", "Quais dificuldades você apresentou no início da implantação do protocolo?", "Você recebeu treinamento da Prefeitura municipal de Florianópolis?", "O treinamento ajudou na utilização do protocolo?", "O que sentiu quando soube que iria prescrever medicações?”, "Sentiu-se bem treinado?”, "Nos últimos seis meses, qual sua média de prescrições medicamentosas para o tratamento das IST?", "Onde você busca material de apoio?", "Me fale sobre sua impressão em relação aos usuários sobre a prática de prescrição medicamentosa realizadas por enfermeiros", "Você se sente satisfeito em realizar essa prática?", "Sugere alguma mudança?".

Todas as entrevistas foram gravadas em meio digital durante as visitas ao local de trabalho dos participantes, conforme agendado anteriormente com cada enfermeiro. Participaram dez deles, respeitandose o critério de saturação e o curto prazo para a coleta de dados. Todos assinaram o Termo de Consentimento Livre e Esclarecido (TCLE) e as entrevistas foram posteriormente transcritas de maneira literal.

A análise dos dados foi realizada conforme a técnica de análise de conteúdo proposto por Bardin, ${ }^{12}$ organizada em torno de três polos cronológicos: leitura compreensiva do material selecionado (sistematização das ideias buscando uma visão de conjunto e a apreensão das particularidades do material); exploração do material (realização de operações para codificação, decomposição dos temas); e elaboração de síntese interpretativa (estabelecimento de quadros que apresentassem as informações de forma articulada aos objetivos da pesquisa e à base teórica). Com base nesse quadro, inferências e interpretações foram realizadas mediante os objetivos propostos. Depois de coletados os dados, as categorias foram construídas com base em leituras extensas e sínteses foram criadas após a interpretação dos resultados.

A pesquisa foi submetida ao Comitê de Ética em Pesquisa com Seres Humanos (CEPSH) sob o Certificado de Apresentação para Apreciação Ética (CAAE) 15390819.4.0000.0118 e aprovada pelo parecer do CEPUDESC 3.672.925. O estudo teve a anuência da Secretaria Municipal de Saúde de Florianópolis. Os participantes tiveram seus nomes substituídos pela sigla $E$ (enfermeiro) junto de uma numeração, conforme a ordem das entrevistas (E1, E2).

\section{RESULTADOS}

Os entrevistados tinham entre 27-57 anos de idade no momento em que responderam aos questionários. Sobre a formação dos dez participantes da pesquisa, destaca-se que todos (100\%) possuem pós-graduação, nove (90\%) são mulheres, oito (80\%) são especialistas em saúde da família e oito (80\%) possuem pelo menos duas especializações. Em relação ao tempo de atuação profissional da amostra selecionada, sete $(70 \%)$ estão há mais de dez anos formados, sete $(70 \%)$ trabalham há pelo menos sete anos na APS do município e todos (100\%) passaram pela capacitação dos protocolos oferecida pela CSAE da Secretaria Municipal de Saúde de Florianópolis. A capacitação relativa ao volume que trata do manejo das IST instrumentalizou os enfermeiros para diagnosticar essas infecções e prescrever o(s) tratamento(s) correspondente(s). Os achados da pesquisa permitem conceber um cenário constituído de um grupo maduro, com formação sólida e devidamente capacitado para atuar clinicamente. 
Os depoimentos após a análise geraram duas categorias: "O enfermeiro e a (re)construção de sua prática profissional" e "O enfermeiro e a sua contribuição na qualidade de serviço na APS".

\section{Categoria 1. 0 enfermeiro e a (re)construção de sua prática profissional}

Nesta primeira categoria, destacam-se palavras como adaptação, suporte e autonomia.

Os enfermeiros referiram que, no início da implementação dos protocolos, sua vida profissional tornou-se permeada por inúmeros sentimentos, sendo a insegurança o mais citado. A maioria dos enfermeiros entrevistados afirmou que a formação da universidade deixou um déficit no que tange à prescrição de medicamentos, mas que, com o passar do tempo, eles souberam lidar com essas mudanças.

Um estudo realizado com enfermeiros que atuam na ESF de um município do estado da Paraíba revelou que poucos se sentiam preparados para a prática da prescrição medicamentosa e apontaram a disciplina de Farmacologia como a responsável pelos resultados da pesquisa. ${ }^{13}$ Algumas falas dos enfermeiros da Secretaria Municipal de Florianópolis entrevistados fazem referência à fragilidade na formação:

"A gente tem o receio pelas questões de farmacologia, que a gente tem certa deficiência na graduação, mas em nenhum momento eu achei que eu não era capaz." (E3)

"A minha formação não foi pra ser prescritor de medicamentos, sou formado pra ser prescritor de cuidados. Fui começando a prescrever devagarinho baseado em alguns protocolos e em estudos de evidência, fui me sentindo mais resolutivo e ao longo do tempo me sentindo seguro." (E4)

"A minha formação foi um pouco diferente da enfermagem que está formando agora. Eu tive um pouco de medo e insegurança. Tive dificuldades, tive que estudar farmacologia de novo. Estudar e a prática ajuda bastante." (E7)

"A minha dificuldade era em relação às interações medicamentosas. Às vezes os pacientes usam alguns medicamentos e eu ficava na dúvida se podia prescrever ou não e eu consultava o médico em relação a isso." (E1)

Apesar de os profissionais do presente estudo relatarem insuficiência na disciplina de Farmacologia, eles mostraram-se dispostos a ampliar suas atribuições. Ainda nesse contexto, podem ser citados como exemplo os enfermeiros da Polônia, que também enfrentaram o mesmo dilema, no entanto expandiram a prática da prescrição medicamentosa nos últimos quatro anos. ${ }^{14}$

Paralelamente ao sentimento negativo de insegurança, os enfermeiros da rede souberam se adaptar ao novo modelo de serviço e citaram a utilização de ferramentas de apoio que dão segurança em suas ações. Alguns entrevistados discorreram sobre a importância de ter um material de pesquisa e de contar com o apoio de outros profissionais para realizarem prescrições medicamentosas:

"Na hora de prescrever eu confiro sinais e sintomas buscando essa informação no protocolo para dar suporte e segurança, uso todas as ferramentas que auxiliam na hora da prescrição. Às vezes, quando me sinto insegura, eu peço o suporte do meu colega médico, então tem sido super tranquilo." (E6)

"O Protocolo de Enfermagem é muito claro, então não há dificuldade de executar por conta dessa clareza, e até porque a gente trabalha com colegas que são mais experientes. Em qualquer momento, ainda que eu tenha um protocolo que me ampare, se eu tenho alguma dúvida, eu recorro a algum enfermeiro que já tinha essa pratica há mais tempo do que eu." (E8)

Os enfermeiros passaram por capacitações que abordaram o tema de prescrição e identificação das IST. Em relação a esses treinamentos, observa-se que alguns dos entrevistados consideraram que não foi fornecido suporte suficiente para a identificação e o tratamento das IST, de modo que eles tiveram de 
buscar recursos adicionais para auxiliá-los. Outros consideraram que o treinamento conseguiu satisfazer suas expectativas:

"O treinamento vem pra dar um start né, ele não consegue em 8 horas de treinamento abordar profundamente todas as questões. O que tu tens que fazer é ler o protocolo e estudar, porque só o treinamento prático ali não consegue dar conta de te deixar totalmente segura pra começar, é preciso se aprofundar com a prática mesmo, com o dia a dia." (E3)

"Todos os enfermeiros que atuam na Estratégia Saúde da Família de Florianópolis recebem treinamento de protocolos, só estamos aptos a usar depois desse treinamento. Eu só tinha leituras e não tinha um treinamento específico, foi um suporte que me deu muita segurança." (E8)

Um estudo realizado no Rio Grande do Norte revelou que enfermeiros mencionam o apoio de outros profissionais e treinamentos/capacitações como elementos centrais para o desenvolvimento de segurança para executarem as prescrições medicamentosas. ${ }^{15}$

Em relação à autonomia, houve um consenso entre os entrevistados de que ela só é atingida pelo enfermeiro quando este profissional executa atividades que realmente domina. Nesse sentido, uma vez que o enfermeiro se enxerga como competente para a identificação e a prescrição medicamentosa no contexto das IST, ele pode efetivamente exercer as suas atribuições com autonomia:

"Esse protocolo foi muito importante para nossa prática por ser algo que a gente tem quase todos os dias, e deu muita autonomia pra esse atendimento. E o protocolo nos guiou para conseguir fazer esse atendimento, a prescrição e o tratamento desses pacientes." (E2)

"Me sinto com autonomia e que eu não preciso a todo momento chamar o auxílio de outro profissional." (E9)

"Me sinto satisfeita em ter maior resolutividade, de poder dar conta da necessidade das pessoas sem ter que acessar outro profissional, e com a possibilidade de aumentar o acesso das pessoas." (E10)

Fica evidente que a autonomia do enfermeiro traz satisfação ao próprio profissional por ampliar seu poder de resolução e, também, por melhorar acesso da população ao serviço de saúde e à qualidade na assistência. Dessa forma, percebe-se que esse processo tende a aprimorar o papel do enfermeiro em seu escopo de atuação na APS, fazendo emergir a segunda categoria.

\section{Categoria 2. O enfermeiro e a sua contribuição na qualidade do serviço na Atenção Primária à Saúde}

Na segunda categoria, destacaram-se termos como satisfação do profissional e satisfação do usuário.

Com os achados da pesquisa, nota-se o enfermeiro com total satisfação relacionada à prática da prescrição medicamentosa. Os protocolos geraram maior grau de independência para a tomada de decisão, e é nítido que os enfermeiros alcançaram seus objetivos de trabalho por meio desse instrumento:

"Eu me senti feliz por poder ajudar os pacientes. O protocolo deu muita autonomia para a gente poder conduzir o caso." (E2)

"Me sinto satisfeita. Não só da prescrição, mas do todo, a gente é mais participativo, nosso atendimento é mais amplo." (E7)

"Isso torna nosso trabalho mais resolutivo, essa autonomia que o enfermeiro tem hoje traz um resultado muito positivo. Os números revelam isso, têm crescido as notificações de IST e a gente logo liga o número de atendimento de enfermagem. Pra gente isso é muito satisfatório." (E8) 
Outro fator de destaque é a visão dos enfermeiros em relação à satisfação do usuário com as consultas de Enfermagem, uma vez que a população por eles assistida procura os serviços públicos de saúde na busca pela resolução de suas queixas:

"Eu tenho uma aceitação de $100 \%$ dos pacientes. Nunca vi recusa de ninguém, nem questionamento, temos sucesso e a aprovação deles de forma bem natural." (E3)

"Os que eu atendi gostaram muito, tiveram resultado eficaz, e não tiveram nenhum problema em ser atendido e prescrito por enfermeiro." (E5)

"De primeiro momento a prescrição de enfermagem é nova para os enfermeiros e usuários. Para o usuário também é uma novidade, mas como ele percebe nossa segurança no atendimento e nossa desenvoltura em trabalhar a explicação, eles aceitam muito bem." (E8)

As prescrições medicamentosas realizadas por enfermeiros visam à excelência de seu trabalho, unind o cuidado e tratamento integrais. Existe uma relação direta entre satisfação e desempenho no trabalho dos enfermeiros e melhoria na saúde dos pacientes. A autonomia no trabalho foi identificada como peça fundamental na satisfação do enfermeiro dada a oportunidade de aplicar todos seus conhecimentos. ${ }^{16}$

Todos os enfermeiros relataram que os usuários se sentem satisfeitos com o atendimento ofertado, dados que corroboram estudos realizados na Espanha e no Canadá. As pesquisas avaliaram de modo positivo a prescrição medicamentosa realizada pelo enfermeiro, comprovando melhora na comunicação entre profissional e usuário, maior informação ao paciente, aumento na frequência de retorno do usuário e melhoria do acesso ao serviço de saúde. Os estudos mostram, ainda, que os usuários apresentam altos índices de satisfação com o atendimento realizado pelo profissional enfermeiro. ${ }^{17,18}$ Percebe-se na fala de uma entrevistada que existe um questionamento por parte do usuário quando este recebe pela primeira vez uma prescrição realizada por um profissional enfermeiro, mas que, com as devidas orientações, o paciente segue o tratamento. Uma pesquisa realizada com usuários da APS no Reino Unido afirmou que os usuários que já tinham conhecimentos prévios sobre a atuação do enfermeiro apresentaram maior satisfação com o atendimento do que aqueles que não conheciam o trabalho desses profissionais. ${ }^{19}$

A prática da prescrição medicamentosa é um componente da consulta de enfermagem e, quando cumprida com competência, tem contribuído para a valorização e a autonomia dos enfermeiros. Quando capacitados, esses profissionais são mais resolutivos, e o usuário confia em sua capacidade clínica. ${ }^{20}$

Não foram relatados pela maioria dos entrevistados pontos negativos nos protocolos ou em sua implantação, ainda que isso não signifique que não existam fragilidades nos materiais em uso e/ou nos treinamentos ofertados. A escassez desses relatos pode ter ocorrido em razão da natureza da pesquisa e do teor/redação das perguntas disparadoras. Outras pesquisas podem ser necessárias para abordar o tema e, até mesmo, fazer contraponto ao presente trabalho.

\section{CONCLUSÃO}

A prática do diagnóstico e da prescrição medicamentosa realizada por enfermeiros amplia o acesso da população aos cuidados de saúde e contribui para o maior reconhecimento profissional da categoria. Tais práticas qualificam ainda mais os cuidados de Enfermagem e aumentam a satisfação do usuário e do próprio profissional com seu trabalho. Assim, o enfermeiro amplia sua prática clínica e adquire autonomia para a tomada de decisão.

Por meio das entrevistas, verificou-se que os enfermeiros se sentem aptos a diagnosticar e prescrever medicamentos para o tratamento das IST, bem como se reconhecem capazes de responder 
adequadamente às necessidades de saúde dos indivíduos. Os participantes declararam-se preparados e seguros para realizar a prescrição. Todavia, não mencionaram a formação acadêmica como a responsável por essa preparação, mas sim a vivência profissional, a facilidade de acesso aos Protocolos de Enfermagem e o auxílio de colegas com mais tempo de prática profissional, provando ser esta uma rede composta de enfermeiros diferenciados. A implantação de protocolos institucionais e a ocorrência de capacitações para a utilização desses instrumentos potencializam a resolubilidade das consultas de enfermagem para os usuários que acessam os serviços da APS do município.

O diagnóstico e a prescrição medicamentosa por enfermeiros são práticas inovadoras de cuidado qualificado, no entanto há escassez de pesquisas nacionais sobre a temática.

\section{AGRADECIMENTOS}

Agradecemos a todos os enfermeiros que colaboraram e cederam entrevistas para as coletas de dados.

\section{CONFLITO DE INTERESSES}

Nada a declarar.

\section{CONTRIBUIÇÕES DOS AUTORES}

BA: Conceituação, Curadoria de Dados, Análise Formal, Escrita - Primeira Redação, Escrita Revisão e Edição. LAP: Conceituação, Curadoria de Dados, Análise Formal, Escrita - Primeira Redação, Escrita - Revisão e Edição. ACSS: Conceituação, Curadoria de Dados, Análise Formal, Escrita - Primeira Redação, Escrita - Revisão e Edição. LNA: Conceituação, Curadoria de Dados, Análise Formal, Escrita Primeira Redação, Escrita - Revisão e Edição. LGP: Conceituação, Curadoria de Dados, Análise Formal, Escrita - Primeira Redação, Escrita - Revisão e Edição. FP: Conceituação, Curadoria de Dados, Análise Formal, Escrita - Primeira Redação, Escrita - Revisão e Edição.

\section{REFERÊNCIAS}

1. World Health Organization (WHO). Sexually transmitted infections: implementing the Global STI Strategy. Genebra: World Health Organization; 2017.

2. Pinto VM, Basso CR, Barros CRDS, Gutierrez EB. Factors associated with sexually transmitted infections: a population based survey in the city of São Paulo, Brazil. Cien Saude Colet 2018;23(7):2423-32. https://doi.org/10.1590/141381232018237.20602016.

3. Brasil. Diretoria de Vigilância Epidemiológica. Boletim epidemiológico HIVIAIDS 2018 Santa Catarina. 2018 [acessado em 13 dez. 2020]. Disponível em: http://www.dive.sc.gov.br/barrigaverde/pdf/BVAidsFINAL2019.pdf

4. Brasil. Boletim Epidemiológico HIVIAIDS/2019. Ministério da Saúde - Secretaria de Vigilância em saúde - Departamento de Doenças de Condições Crônicas e Infecções Sexualmente Transmissíveis - DCCI. 2019[acessado em 23 dez. 2020]. Disponível em: http://www.aids.gov.br/pt-br/pub/2019/boletim-epidemiologico-de-hivaids-2019

5. Barbosa TLA, Gomes LMX, Holzmann APF, Paula AMB, Haikal DSA. Aconselhamento em doenças sexualmente transmissíveis na atenção primária: percepção e prática profissional. Acta Paul Enferm 2015;28(6):531-8. http://doi. org/10.1590/1982-0194201500089

6. Bungay V, Masaro CL, Gilbert M. Examining the scope of public health nursing practice in sexually transmitted infection prevention and management: what do nurses do? J Clin Nurs 2014;23(21-22):3274-85. http://doi.org/10.1111/jocn.12578

7. Brasil. Decreto- Lei $n^{\circ} 7.498$, de 25 de junho de 1986. Dispõe sobre a regulamentação do exercício da Enfermagem e dá outras providências [Internet].1986. [acessado em 10 jan. 2021]. Disponível em: http://www.cofen.gov.br/lei-n-749886-de25-de-junho-de-1986_4161.html 
8. Brasil. Ministério da Saúde. Portaria n 2.436, de 21 de setembro de 2017. Aprova a Política Nacional de Atenção Básica, estabelecendo a revisão de diretrizes para a organização da Atenção Básica, no âmbito do Sistema Único de Saúde (SUS) [Internet]. 2017. [acessado em 13 dez. 2020]. Disponível em: https://bvsms.saude.gov.br/bvs/saudelegis/gm/2017/ prt2436_22_09_2017.html

9. Ferreira IT, Neves KTQ, Oliveira AWN, Galvão TRAF, Mangane EM, Sousa LB. Avaliação da qualidade da consulta de enfermagem em infecções sexualmente transmissíveis. Enferm Foco (Brasília) 2018;9(3):42-7. http://doi.org/10.21675/2357707X.2018.v9.n3.1119

10. Florianópolis. Secretaria Municipal de Saúde. Portaria/SS/GAB/No 283/2007. Aprova a Política Municipal de Atenção à Saúde, estabelecendo diretrizes e normas para a organização da Atenção Básica baseada na Estratégia de Saúde da Família. Florianópolis - SC, 2007[Internet]. 2007 [acessado em 14 jan. 2021]. Disponível em: http://www.pmf.sc.gov.br/ entidades/saude/index.php?cms $=$ saude+da+familia\&menu=5

11. Florianópolis. Secretaria Municipal de Saúde. Portaria No 79/2015. Institui a Comissão Permanente de Sistematização da Assistência de Enfermagem - CSAE [internet]. 2015 [acessado em 14 jan. 2021]. Disponível em: http://www.pmf.sc.gov.br/ arquivos/arquivos/pdf/05_08_2015_14.01.47.1db139dd6a2842c9796b6345c54e03e8.pdf

12. Bardin L. Análise de conteúdo. São Paulo: Edições 70; 2011. 229 p.

13. Martiniano CS, Marcolino EC, Souza MB, Coelho AA, Arcêncio RA, Fronteira I, et al. The gap between training and practice of prescribing of drugs by nurses in the primary health care: a case study in Brazil. Nurse Educ Today 2016;36:304-9. http:// doi.org/10.1016/j.nedt.2015.07.017

14. Maier CB. Nurse prescribing of medicines in 13 European countries. Hum Resour Health 2019;17(1):95. http://doi.org/10.1186/ s12960-019-0429-6

15. Vasconcelos RB, Araújo JL. A prescrição de medicamentos pelos enfermeiros na Estratégia Saúde da Família. Cogitare Enferm 2013;18(4):743-50. http://doi.org/10.5380/ce.v18i4.34931

16. Giles M, Parker V, Mitchell R, Conway J. How do nurse consultant job characteristics impact on job satisfaction? An Australian quantitative study. BMC Nurs 2017;16:51. http://doi.org/10.1186/s12912-017-0246-y

17. Sánchez-Gómez MB, Ramos-Santana S, Gómez-Salgado J, Sánchez-Nicolás F, Moreno-Garriga C, Duarte-Clíments G. Benefits of advanced practice nursing for its expansion in the spanish context. Int J Environ Res Public Health 2019;16(5):680. http://doi.org/10.3390/ijerph16050680

18. Woo BFY, Lee JXY, Tam WWS. The impact of the advanced practice nursing role on quality of care, clinical outcomes, patient satisfaction, and cost in the emergency and critical care settings: a systematic review. Hum Resour Health 2017;15(1):63. http://doi.org/10.1186/s12960-017-0237-9

19. Barratt J, Thomas N. Nurse practitioner consultations in primary health care: a case study-based survey of patients' preconsultation expectations, and post-consultation satisfaction and enablement. Prim Health Care Res Dev 2018;20:e36. http://doi.org/10.1017/S1463423618000415

20. Bellaguarda MLR, Nelson S, Padilha MI, Caravaca-Morera JA. Autoridade Prescritiva e Enfermagem: uma análise comparativa no Brasil e no Canadá. Rev Latino-Am Enfermagem 2015;23(6):1065-73. http://doi.org/10.1590/0104-1169.0418.2650 